\title{
Crystallization of Amorphous Silicon Films with Native-Oxide Free Surfaces
}

\author{
Akira SAKaI*, Toru TatSUMI, Taeko NinNo, Haruhiko ONO and Koichi ISHIDA
}

Received August 13, 1991; Accepted September 17, 1991

\begin{abstract}
Crystallization of amorphous $\mathrm{Si}$ (a-Si) on $\mathrm{SiO}_{2}$ layers under ultra-high vacuum (UHV) conditions was examined. In the case that the a-Si layer with a native oxide free surface was annealed in the UHV molecular-beam-epitaxy chamber, it has been found that the initial nucleation and the grain growth occur at the surface. Cross-sectional transmission electron microscopy revealed the novel mode of the grain growth which resulted in the formation of mushroom-shaped Si grains at the a-Si surface. The surface crystallization phenomenon was found to be sensitive to the oxygen partial pressure during the annealing. Reappearance of the surface crystallization was also confirmed in the air-exposed a-Si film whose surface native-oxide layer was removed by HF solution just before the UHV annealing. The crystallization mechanism of the a-Si with the clean surface was discussed.
\end{abstract}

\section{INTRODUCTION}

In recent $\mathrm{Si}$ electric device technology, polycrystalline $\mathrm{Si}$ (poly-Si) films are widely used for electrodes. The electrical performance of the poly-Si electrode is mostly determined by its texture, grain size, and surface morphology. Full understanding of the growth mechanism of the poly-Si is required for controlling such factors. Solid phase growth (SPG) of amorphous $\mathrm{Si}(\mathrm{a}-\mathrm{Si})$ on a $\mathrm{SiO}_{2}$ layer is one of the formation techniques of the poly-Si layers and has been carried out by using various methods, generally as a part of the silicon-on-insulator technology. A conventional technique of the a-Si SPG consists of two procedures; the fabrication of the a-Si layer by (low pressure: LP) chemical vapor deposition $(\mathrm{CVD})^{1,2)}$, deposition by ultra-high vacuum (UHV) evaporation $^{3 \sim 6)}$, or self-implantation ${ }^{7 \sim 9)}$, and the annealing for crystallization of the a-Si in vacuum or an inert atmosphere, such as $\mathrm{Ar}$ or $\mathrm{N}_{2}$.

Microelectronics Research Laboratories, NEC Corporation, (34 Miyukigaoka, Tsukuba, Ibaraki 305, Japan)

Key Words: Amorphous Silicon, Crystallization, Native Oxide Layer, Surface, Nucleation, HF Treatment, $\mathrm{O}_{2}$ Partial Pressure
It should be noticed that a surface of the a-Si is subjected to native oxidation before the annealing in the conventional methods. Up to now, there are few studies of SPG of a-Si films without native oxide layers on their surfaces, although the measurement of the crystallization time for the UHV-deposited a-Si film annealed under UHV conditions was carried out by Olson et a $l^{10}$.

In this paper, we will report the experimental results of $\mathrm{SPG}$ of the a-Si on the $\mathrm{SiO}_{2}$ layers under $\mathrm{UHV}$ conditions. In the case that a-Si layer deposition on the $\mathrm{SiO}_{2}$ layers and annealing for crystallization were successively carried out in a UHV molecular-beam-epitaxy (MBE) chamber, we found for the first time a novel mode of crystallization which is completely different from that observed previously. It consists of the nucleation at the surface of the a-Si and the formation of mushroom-shaped Si grains, suggesting high mobility of atoms on the a-Si clean surface ${ }^{11)}$. We also investigated the effect of the $\mathrm{O}_{2}$ partial pressure during the annealing on the growth mode. Furthermore, crystallization of the air-exposed a-Si film whose surface native 
oxide layer was removed by HF solution, was examined under the UHV condition.

\section{EXPERIMENTAL}

The a-Si deposition and the annealing for crystallization were performed by using MBE equipment (base pressure: $1 \times 10^{-10}$ Torr) which allows $\mathrm{O}_{2}$ and $\mathrm{N}_{2}$ gases to be introduced. The a-Si layers of $500 \sim 1000 \AA$ thicknesses were formed on thermal oxide layers on $\mathrm{Si}(001)$ wafers by $\mathrm{Si}$ molecular beam deposition at room temperature at a rate of $7 \AA /$ sec. The silicon source was an electron beam evaporator $(6 \mathrm{kV})$. The annealing temperature was varied from 500 to $700^{\circ} \mathrm{C}$. Heating rates of the samples were also varied in the range $1 \sim 10^{\circ} \mathrm{C} / \mathrm{sec}$. The variation of a-Si surface crystallinity during the annealing was monitored, in situ, by reflection high energy electron diffraction (RHEED) observation, in which the RHEED halo pattern of the amorphous layers turned into a polycrystalline ring one. Surface morphology of the crystallized a-Si was observed by scanning electron microscopy (SEM). Detail structures were examined by transmission electron microscopy (TEM). Auger electron spectroscopy (AES) and secondary ion mass spectrometry (SIMS) were also employed.

\section{RESULTS}

\subsection{Structure of crystallized a-Si in UHV}

Figure 1(a) and (b) are typical surface SEM images of the a-Si film, which were subsequently annealed in UHV just after the deposition at $600^{\circ} \mathrm{C}$ for 5 and $10 \mathrm{~min}$, respectively. Many protrusions can be seen on a flat surface in Fig. 1(a) and the density of the protrusion increases for a longer annealing time so that the uneven surface, as shown in Fig. 1(b), is formed. Figure 2 is a cross-sectional high-resolution (HR) TEM image of the sample shown in Fig. 1(a). The protrusion is revealed to the Si grain, in which \{111\} lattice fringes are mainly observed. However, the region marked A shows a mottled contrast indicating an amorphous state in the layer. These results confirm that the initial nucleation for crystallization does not occur at the a

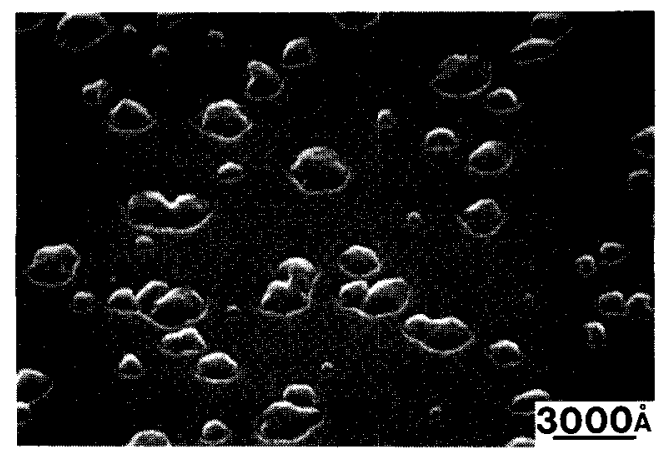

b

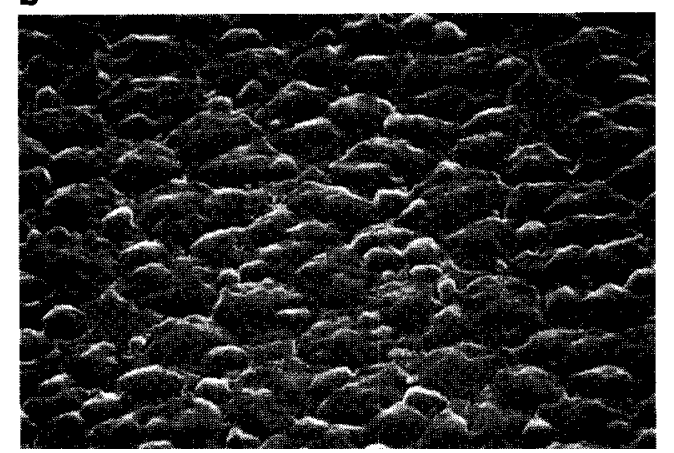

Fig. 1 SEM images showing surface morphology of a-Si film surfaces annealed at $600^{\circ} \mathrm{C}$ for (a) $5 \mathrm{~min}$ and (b) $15 \mathrm{~min}$.

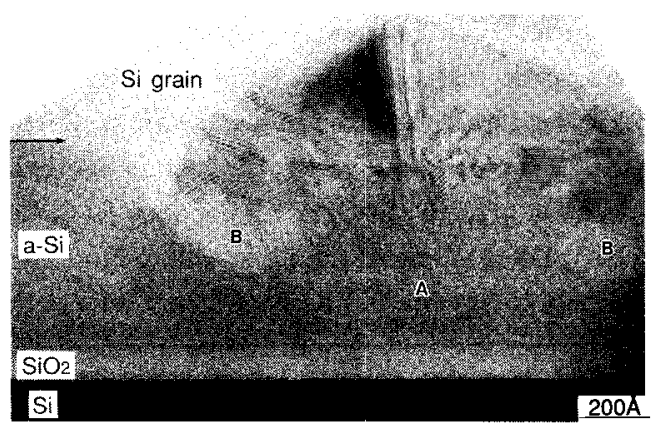

Fig. 2 A cross-sectional TEM micrograph showing the vicinity of the $\mathrm{Si}$ grain. A region shows the amorphous state under the $\mathrm{Si}$ grain. B regions indicate the depletions of the a-Si layer. The arrow shows the original position of the a-Si surface.

a-Si/ $/ \mathrm{SiO}_{2}$ interface but in the vicinity of the a-Si surface. It is also notable in Fig. 2 that the preferential depletion of the a-Si around the $\mathrm{Si}$ grain can be seen in the region marked B. Furthermore, the top of the Si grain is located above the original position of the a-Si surface indicated 
by an arrow and thus the whole feature resembles a mushroom shape. These results clearly show that mass transfer has occurred from the a-Si layer to the Si grain during annealing.

Further observations were carried out for various types of samples, which were annealed at different temperatures and time, and which had different thicknesses of a-Si and $\mathrm{SiO}_{2}$ layers. Samples having other types of $\mathrm{SiO}_{2}$ layers were also observed; a $\mathrm{SiO}_{\mathbf{x}}(\mathrm{x} \sim 2)$ layer formed using an oxygen molecular beam ${ }^{12)}$ and $\mathrm{a} \mathrm{SiO}_{2}$ layer formed using ECR oxygen plasma. All results showed that the nucleation always occurred at the a-Si surface and the mushroom-shaped grains were formed. Thus the phenomenon of the surface nucleation and the grain growth on the a-Si surface is found to be independent of annealing conditions, structure of deposited layers, and the $\mathrm{SiO}_{2}$ layer underneath the a-Si.

Next, in order to examine the influence of the surface cleanliness on the nucleation site and the grain growth, samples whose surfaces were covered with native oxide layers were investigated. After the a-Si deposition on the $\mathrm{SiO}_{2}$ layer, the sample was removed from the MBE chamber and exposed to air for several days. With this procedure, it was expected that the $\mathrm{Si}$ atoms in the surface region of several angstroms would be passivated by the native oxide layer. The sample was then loaded into the MBE chamber again and annealed in UHV. For the samples prepared under various annealing conditions, no pattern changes in the RHEED observation were seen, i.e., the amorphous halo pattern persisted during the annealing. Therefore, in this case, the initial crystallization at the a-Si surface was not detected by the RHEED observation. Figure 3 is a cross-sectional TEM image of the sample annealed at about $600^{\circ} \mathrm{C}$ for $60 \mathrm{~min}$, showing the $\mathrm{Si}$ grains embedded in the a-Si layer. No mushroom-shaped $\mathrm{Si}$ grains can be observed and the surface morphology is rather flat. The nucleation appeared to occur in the vicinity of the a-Si/ $/ \mathrm{SiO}_{2}$ interface. These results demonstrate that the surface nucleation occurs only for the a-Si layer with a clean surface.

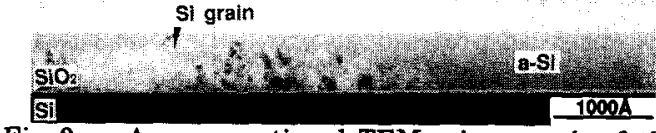

Fig. 3 A cross-sectional TEM micrograph of the sample, whose surface was exposed to air just before the UHV annealing.

\subsection{Effect of $\mathrm{O}_{2}$ on surface crystallization}

Next we investigate the effect of $\mathrm{O}_{2}$ partial pressure on the surface crystallization of a-Si films. After the deposition of a-Si, a $99.9999 \% \mathrm{O}_{2}$ gas was introduced into the MBE chamber to the designated pressure and then the wafer was annealed at $650^{\circ} \mathrm{C}$ for $5 \mathrm{~min}$. Figure $4(\mathrm{a})$ and (b) show SEM images of the surface morphology and the corresponding AES profiles of the crystallized a-Si surface, respectively, at respective $\mathrm{O}_{2}$ partial pressures. Under the partial pressure of $2 \times 10^{-6}$ Torr, uneven surface morphology and the only $\mathrm{Si}$ peak in AES profile are observed. On the other hand, flat surfaces and oxygen peaks can be seen in the samples annealed under the $\mathrm{O}_{2}$ partial pressures of $2 \times 10^{-5}$ and $2 \times 10^{-4}$ Torr. The chemical shift of the $\mathrm{Si}$ peak to $\mathrm{SiO}_{2}$ are also observed in the both samples. These results show that the oxide layer are formed on the a-Si surface during the annealing under higher $\mathrm{O}_{2}$ partial pressures. As a consequence, no surface crystallization has occurred leading to the formation of the flat surface morphology. Furthermore, we have confirmed that the introduction of a $\mathrm{N}_{2}$ gas into the MBE chamber during the annealing has no influences on the surface crystallization.

\subsection{Effect of HF-treatment on surface crystallization}

The above results clearly indicate that the oxide layer formed on the a-Si surface inhibited the surface crystallization. Thus we tested the HF-treatment, which is conventionally used to remove thin oxide layers from the wafer surface by $\mathrm{HF}$ solution ( $\mathrm{HF}: \mathrm{H}_{2} \mathrm{O}=1: 30$ ), for the native oxide-covered a-Si film. After exposure of a wafer with a UHV-deposited a-Si film into air, the wafer was dipped into the HF solution for $1 \mathrm{~min}$. Then it was loaded into the MBE chamber again and annealed at $650^{\circ} \mathrm{C}$ for $5 \mathrm{~min}$. Figure $5(\mathrm{a})$ is a 
SEM image showing the surface morphology. The observed protrusions prove that the surface crystallization did occur in the sample. However, the region of the flat surface can be also seen in spite of the sufficient annealing temperature and time to crystallize all a-Si region. As shown in Fig. 5(b), a cross-sectional TEM image reveals that this sample consists of polycrystalline regions with flat surfaces and protrusive grains. It is also notable that a wedge-shaped concavity, as indicated by an arrow, is observed around the grain.
In the same way, we also applied the HFtreatment to the air exposed a-Si films fabricated by LPCVD. Surface morphology of the LPCVD sample, which was annealed in UHV after the HF-treatment, is shown in Fig. 6. In this case, the protrusive $\mathrm{Si}$ grains are observed on the whole surface, forming uneven surface morphology which is similar to that in Fig. 1(b). To verify the substantial differences in the crystallization modes between the a-Si films formed by MBE (MBE a-Si) and by LPCVD (LPCVD a-Si), distribution of impurities $(\mathrm{H}, \mathrm{O}, \mathrm{C}, \mathrm{N})$ was analyzed

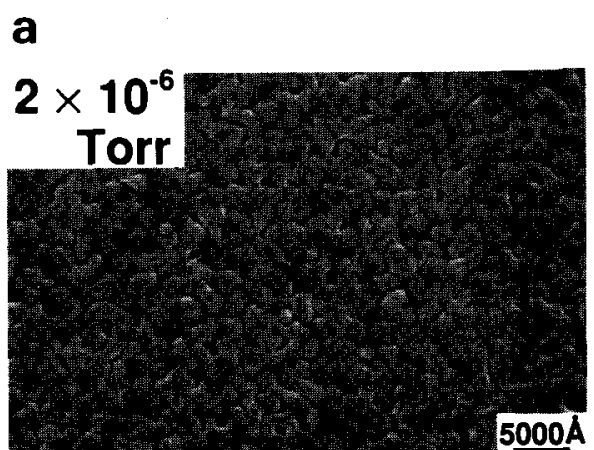

b
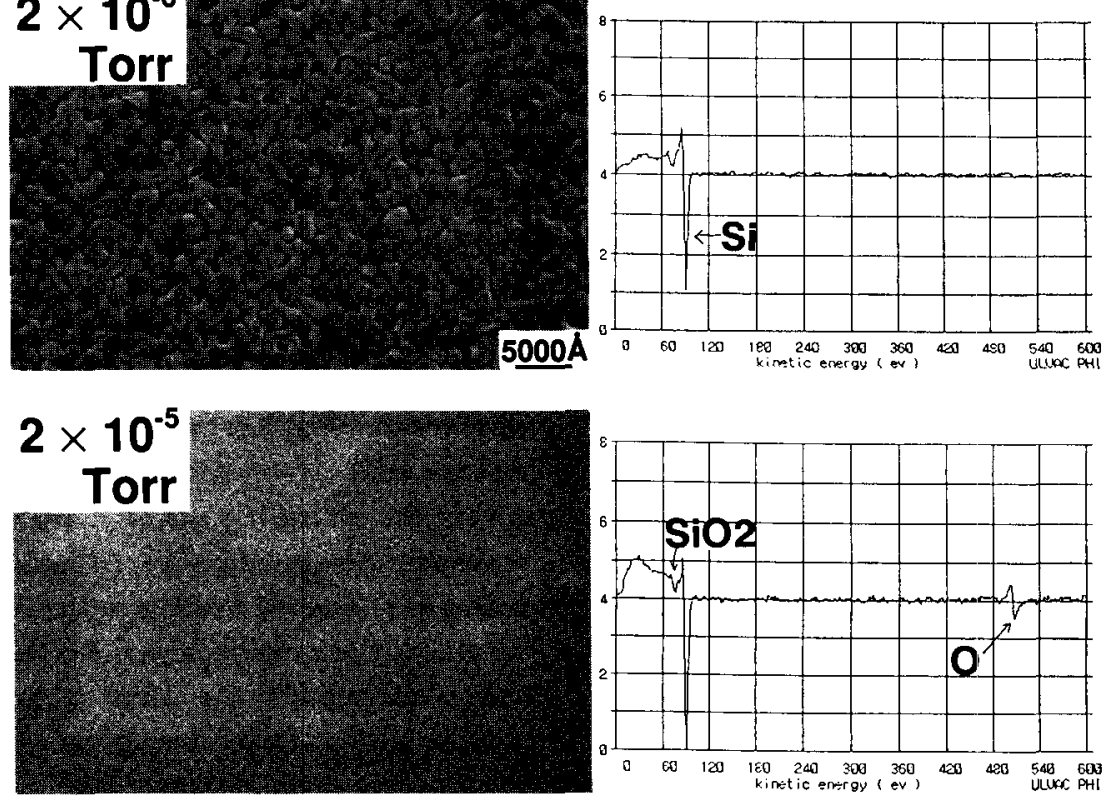

\section{$2 \times 10^{-4}$ Torr}
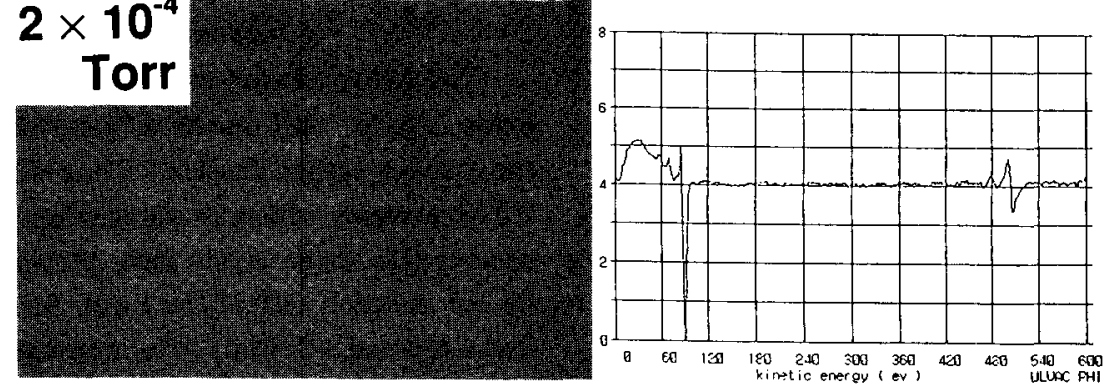

Fig. 4 Dependence of surface morphology (a) and surface AES profiles (b) upon $\mathrm{O}_{2}$ partial pressures of $2 \times 10^{-6}, 2 \times 10^{-5}$, and $2 \times 10^{-4}$ Torr. 


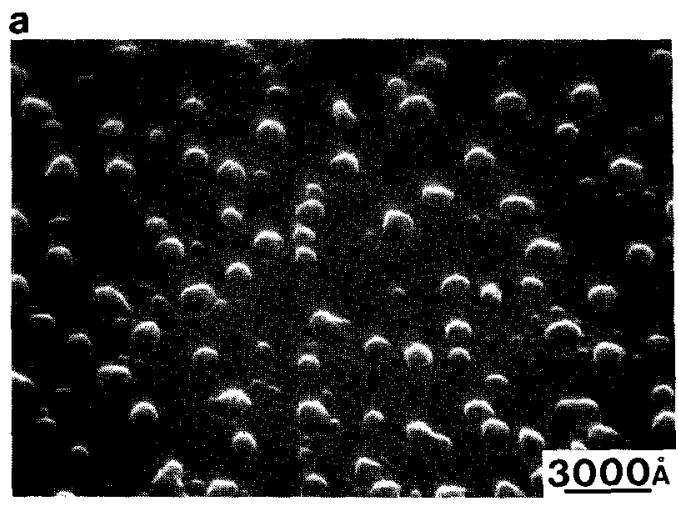

b

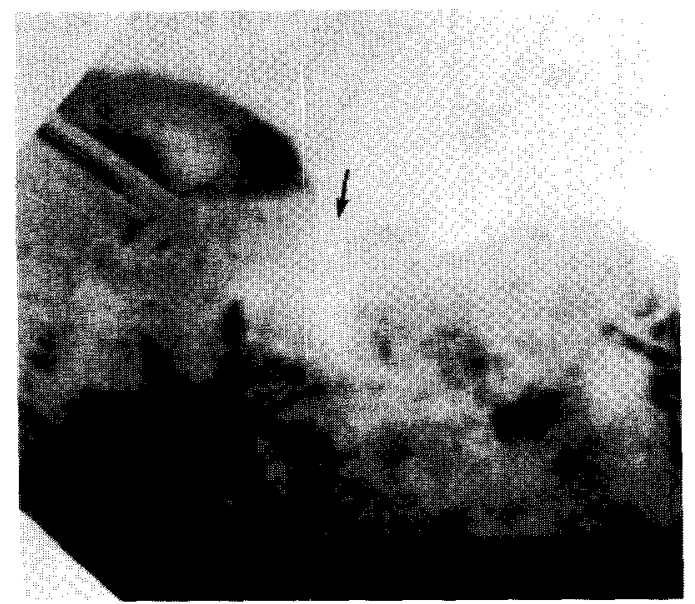

Fig. 5 (a) A surface SEM image of the MBE a-Si film annealed at $650^{\circ} \mathrm{C}$ for $5 \mathrm{~min}$, whose native oxide layer was removed by HF solution before the UHVannealing.

(b) A cross-sectional TE'M micrograph of the vicinity of the protrusion shown in (a).

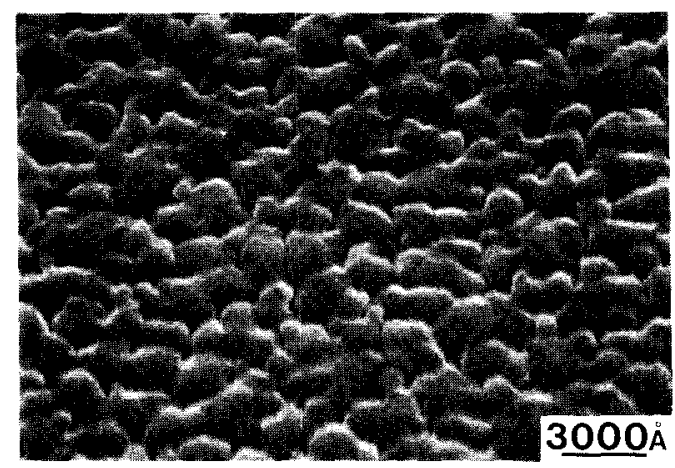

Fig. 6 A surface SEM image of the LPCVD a-Si film annealed at $650^{\circ} \mathrm{C}$ for $5 \mathrm{~min}$, whose native oxide layer was removed by $\mathrm{HF}$ solution before the $\mathrm{UHV}$ annealing. by SIMS. Figure 7 shows the concentration profiles of respective impurities in the samples which were dipped into $H F$ solution. Note that the concentration of $\mathrm{H}, \mathrm{O}$, and $\mathrm{N}$ in MBE a-Si are higher than that in LPCVD a-Si. Especially, the profiles of $\mathrm{H}$ and $\mathrm{O}$ in $\mathrm{MBE}$ a-Si suggest the percolation of $\mathrm{H}_{2} \mathrm{O}$ molecule into the film.

\section{DISCUSSION}

We can now deduce a mechanism of SPG of a$\mathrm{Si}$ under the UHV condition. In case of the conventional SPG, it is believed that the nucleation at the a-Si/ $/ \mathrm{SiO}_{2}$ interface is due to the release of the stress induced by the thermal expansion difference between $\mathrm{Si}$ and $\mathrm{SiO}_{2}{ }^{2,6)}$ and/or of that which is attributed to the porosity of the a-Si, especially that deposited by vacuum-evaporation at room temperature ${ }^{13)}$. However, such a stress relaxation mechanism can not explain the surface nucleation phenomenon as in the present case. Thus we require another mechanism. First, compared to the crystallization time of the native oxide covered a-Si, that of the a-Si with clean surfaces is shorter by a factor of 10 . This result suggests high mobility $\mathrm{f} \mathrm{Si}$ atoms in a-Si, which contribute to crystallization. Second, the preferential depletions of the a-Si around the $\mathrm{Si}$ grains shown in Fig. 2 directly indicate the migration of $\mathrm{Si}$ atoms around the nucleus. Third, such phenomenon is very sensitive to the surface cleanliness of a-Si. Therefore, we can propose one possible mechanism of SPG of a-Si under the UHV condition as follows (see Fig. 8). It is deduced that the mobility of $\mathrm{Si}$ atoms on the clean a-Si surface is high enough to contribute to the nucleation and the grain growth at the surface (Fig. 8(a)). If the surface atoms could migrate with high mobility near the temperature of the aSi crystallization, the migrating atoms would collide with high frequency and then nucleate at the a-Si surface (Fig. 8(b)). The nucleation rate at the surface may be higher than that at the interface, at least in the temperature range of the experiment. The grain growth can proceed in two ways. One is a conventional solid phase growth from the nucleus downward into the a-Si. The 
a

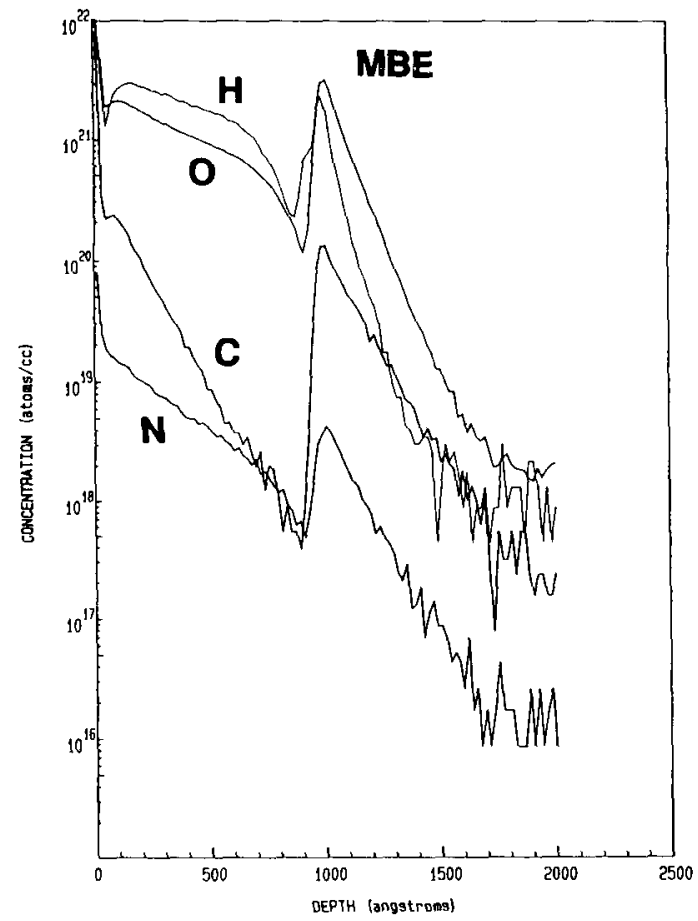

b

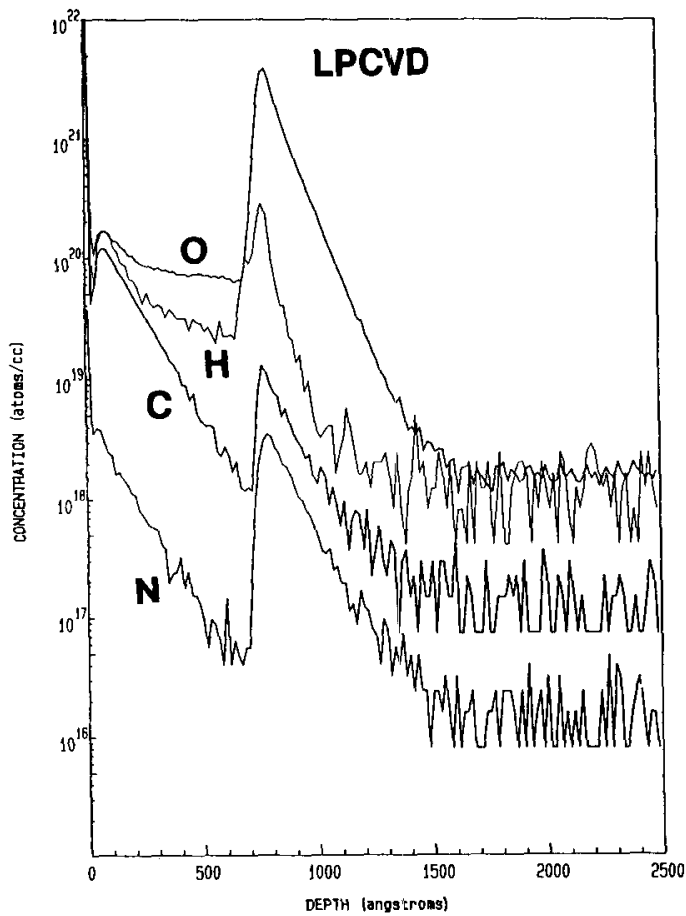

Fig. 7 SIMS analyses of $\mathrm{H}, \mathrm{O}, \mathrm{N}$, and $\mathrm{C}$ in the $\mathrm{MBE}$ (a) and LPCVD (b) a-Si films just after the HF treatment.

a

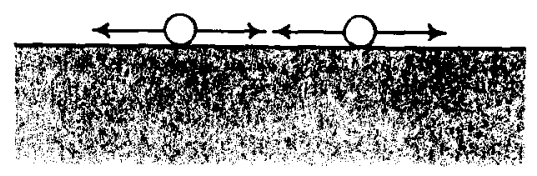

C

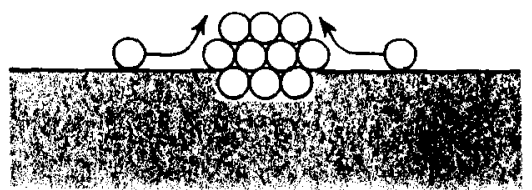

b

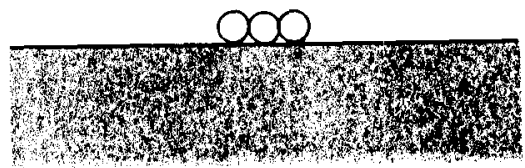

d

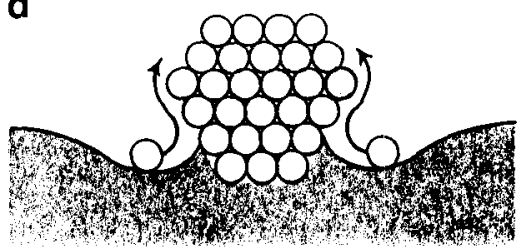

Fig. 8 Model of crystallization of the a-Si film with a clean surface.

other is a novel mode in which the migrating atoms with high mobility climb up the surface of the nucleus (Fig. 8(c)). The nucleus virtually acts a sink of such surface migrating atoms and grows into the Si grain (Fig. 8(d)).

It was previously reported that the electron evaporated a-Si film was easily subjected to the gas absorption $^{14)}$. The gas species, such as $\mathrm{O}$ and
$\mathrm{H}_{2} \mathrm{O}$ in this case, are considered to inhibit the atom migration partially on the a-Si surface during the annealing. This means that the supply of $\mathrm{Si}$ atoms which will be crystallized is also restricted to the region where surface crystallization can occur. This idea well explains the structure of the wedge shaped concavity around the protrusive grain observed in Fig. 5(b). 
Recently, it has been reported by Watanabe et al. that poly-Si layers, which have uneven surface morphology composed of hemispherical grains, are grown by LPCVD in specific growth conditions ${ }^{15}$. The poly-Si was successfully applied to storage electrodes of stacked capacitors of dynamic-random-access-memories. Since the structures of the poly-Si layers are quite similar to our case, it is suggested that the crystallization with the above mentioned mechanism can also occur in the LPCVD process. Furthermore, reappearance of the surface crystallization was also confirmed in the air-exposed LPCVD a-Si film whose surface native-oxide layer was removed by HF solution just before the UHV annealing. Therefore, the surface crystallization phenomenon is substantially dependent on cleanliness of the surface and independent of the fabrication method of the a-Si film. It is important to suppress the oxygen partial pressure during the a-Si fabrication and the subsequent annealing in order to accomplish the present type of crystallization.

\section{CONCLUSION}

Crystallization of the a-Si films was performed under the UHV condition. The experimental results showed the novel crystallization mode suggesting high mobility of surface atoms of a-Si, which led to the formation of uneven surface morphology. It is pointed out that this phenomenon belongs to the category of the crystallization of a-Si films which have clean surfaces.

The authors would like to thank H.Watanabe and N. Ikarashi for their useful discussions.
Thanks are also due to Y. Matsumoto, M. Nakamae, M. Ogawa. H. Watanabe, and F.Saito for their encouragement.

\section{REFERENCES}

1) R. Bisaro, J. Magariño, K. Zellama, S. Squelard, P. Germain, and J.F. Morhange, Phys. Rev., B31, 3568(1985).

2) E. Adachi, T. Aoyama, N. Konishi, T. Suzuki, Y. Okajima, and K. Miyata, Jpn. J. Appl. Phys., 27, L1809(1988).

3) K. Zellama, P. Germain, S. Squelard, J. C. Bourgoin, and P. A. Thomas, J. Appl. Phys., 50, 6995(1979).

4) H. Yamamoto, H. Ishiwara, and S. Furukawa, Jpn.J. Appl.Phys., 24, 411(1985).

5) Y. Kunii and M. Tabe, Jpn. J. Appl. Phys., 24, L352(1985).

6) M. Moniwa, M. Miyao, R. Tsuchiyama, A. Ishizaka, M. Ichikawa, H. Sunami, and T. Tokuyama, Appl.Phys. Lett., 47, 113(1985).

7) Y. Ohyama, Y. Matsushita, and M. Kashiwagi, Jpn.J.Appl. Phys., 21, L152(1982).

8) R. B. Iverson and R. Reif, J. Appl. Phys., 62, 1675(1987).

9) A. Nakamura, F. Emoto, E. Fujii, Y. Uemoto, A. Yamamoto, K. Senda, and G. Kano, Jpn.J. Appl.Phys., 27, L2408(1988).

10) G. L. Olson and J. A. Roth, Materials Science Reports, 3, 1(1988).

11) A. Sakai, H. Ono, K. Ishida, T. Niino, and T. Tatsumi, Jpn. J. Appl. Phys., 30, L941 (1991).

12) T. Tatsumi, T. Niino, A. Sakai, and $H$. Hirayama, Jpn. J. Appl. Phys., 28, L1678 (1989).

13) H. Ishiwara, H. Yamamoto, S. Furukawa, M. Tamura, and T. Tokuyama, Appl. Phys. Lett., 43, 1028(1983).

14) J. C. Bean and J. M. Poate, Appl. Phys. Lett., $36,59(1980)$.

15) H. Watanabe, N. Aoto, S. Adachi, T. Ishijima, E. Ikawa, and K. Terada, Appl. Phys. Lett., 58, 251(1991). 